\title{
INTRODUÇÃO E DESENVOLVIMENTO DO USO DA COMUNICAÇÃO ALTERNATIVA NA SÍNDROME DE ANGELMAN: ESTUDO DE CASO
}

\author{
Introduction and developing alternative communication \\ in syndrome of Angelman: case study
}

\author{
Melissa Watzko Eskelsen (1), Fernanda Bernadeth Pacheco (2), Cristiane Gonçalves Montibeller (3), \\ Helena Ferro Blasi ${ }^{(4)}$, Raquel Fleig ${ }^{(5)}$
}

\begin{abstract}
RESUMO
Tema: Síndrome de Angelman (SA) e comunicação alternativa. Procedimentos: com o objetivo de descrever o processo de introdução e desenvolvimento do uso do Sistema Alternativo de Comunicação (SAC) na SA. Foi utilizado um estudo de caso, realizado na Clínica de Fonoaudiologia da Faculdade Estácio de Sá de Santa Catarina. E., diagnosticada com SA, 17 anos, sem linguagem oral. A coleta de dados foi realizada com 8 trechos de terapias filmadas durante o primeiro semestre de 2007 e anotações no diário de campo. Resultados: o programa de comunicação escolhido foi o Picture Communication Symbols System (PCS). Para seleção dos símbolos, foi utilizado centros de interesse da paciente. O processo de inserção de símbolos aconteceu de maneira lenta e gradativa. A terapeuta realizava a apresentação do objeto concreto, em seguida, correlacionava o símbolo com o objeto. Os dois eram postos em cima da mesa, correlacionandoos várias vezes. Para introdução da paciente ao uso do sistema a terapeuta pegava a mão da paciente e colocava sobre o símbolo e simultaneamente entregava o objeto. Com o passar das sessões foram introduzidos novos símbolos. E. mostrou-se "perdida" negando categorias apresentadas, direcionando sua escolha para os símbolos que já lhe eram familiar. Dos 15 símbolos trabalhados, adquiriu funcionalidade para 5 símbolos. Conclusão: o SAC torna-se um meio viável de comunicação para SA. O processo de introdução e desenvolvimento de uso de SAC foi composto pela: apresentação, correlação e introdução da paciente ao uso do sistema. Salienta-se que é um trabalho lento que exige participação da família.
\end{abstract}

DESCRITORES: Síndrome de Angelman; Comunicação; Linguagem

(1) Fonoaudióloga; Docente do Curso de Fonoaudiologia da Faculdade Estácio de Sá de Santa Catarina, FESSC, São José, SC; Curso de Especiação em Reabilitação Neurológica, Saúde Pública e Educação Especial com Práticas Inclusivas; Mestre em Saúde Pública.

(2) Fonoaudióloga.

(3) Fonoaudióloga; Docente do Curso de Fonoaudiologia da Faculdade Estácio de Sá de Santa Catarina, FESSC, São José, SC; Mestre em Neurociências.

(4) Fonoaudióloga; Docente e Coordenadora do Curso de Fonoaudiologia da Faculdade Estácio de Sá de Santa Catarina, FESSC, São José, SC; Doutora em Linguística.

(5) Fonoaudióloga; Docente do Curso de Fonoaudiologia da Faculdade Estácio de Sá de Santa Catarina, FESSC, São José, SC; Especialista em Psicopedagogia; Mestre em Engenharia de Produção.

Conflito de interesse: INEXISTENTE

\section{INTRODUÇÃO}

A Síndrome de Angelman (SA) é uma doença de base genética, rara, caracterizada por alterações como retardo mental severo, língua em protrusão, risadas sem motivos, movimentos atáxicos e bruscos, microcefalia, convulsões, alteração na aquisição e desenvolvimento da linguagem, capacidade de fala mínima ou nula, entre outros ${ }^{1,2}$. Foi descoberta em 1965 por Harry Angelman 3,4.

Outros estudos referiram retardo mental severo, afasia e epilepsia ${ }^{4}$. Outro autor acrescenta características físicas e hiperatividade ${ }^{3}$.

A incidência descrita na literatura apresenta diferença entre os números apresentados, sendo 
de 1 (um) caso para 12.000 (doze mil) habitantes a 15.000 (quinze mil) ${ }^{5}$.

As principais características da SA são: retardo mental severo, alteração na aquisição da linguagem, características faciais típicas da síndrome ${ }^{6}$. Quando a criança nasce, apresenta características normais, com um a dois meses começa a ter grande dificuldade em se alimentar. O perímetro encefálico se desenvolve abaixo de crianças normais. No desenvolvimento motor ocorre atraso e a criança consegue andar somente após três anos de vida. Há atraso também na aquisição de linguagem. Além disso, a criança está sempre sorrindo e apresenta hiperatividade ${ }^{4}$.

O fonoaudiólogo é responsável pelo estudo da comunicação humana pesquisando métodos e técnicas de prevenção e terapia, realizadas na comunicação oral, escrita, voz e audição ${ }^{7}$. "A comunicação permita ao homem transmitir e interpretar sentimentos e pensamentos, interagindo com outros indivíduos numa troca de informações e experiências

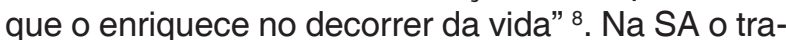
balho fonoaudiológico prioriza além da adequação da motricidade oromiofuncional, o desenvolvimento da comunicação seja ela verbal ou não verbal.

As alterações de linguagem existentes podem ser primárias, como o distúrbio específico de linguagem, ou secundárias ${ }^{9}$, como na SA. Tendo em vista que uma das principais características secundárias da SA é a ausência da fala funcional, sugere-se a introdução e desenvolvimento de Sistemas Alternativos de Comunicação (SAC) para proporcionar ao portador desta síndrome um meio de comunicação mais efetivo com o mundo exterior. "Trata-se de uma forma extraordinária de desenvolvimento linguístico, mas o objetivo final seria que as crianças que estejam desenvolvendo modos alternativos de comunicação sejam capazes de se comunicar com seus pares e com os adultos sobre os mesmos assuntos e nas mesmas situações que as crianças falantes" ${ }^{10}$.

O enfoque predominante do trabalho realizado é centrar a atenção na pessoa proporcionando oportunidade de inclusão social e educacional. Considera-se a possibilidade de alternativas e práticas que promovam a participação e desenvolvimento integral da pessoa portadora de necessidades especiais ${ }^{11}$, ou seja, pensar na diversidade ${ }^{12}$, fazendo com que a individualização consista no respeito às características de cada pessoa ${ }^{13,14}$.

Existem vários SAC como: Blissynbolics System (BLISS), Pictogram Ideogram Communication System (PIC) e Picture Communication Symbols System (PCS), ficando a critério do profissional de Fonoaudiologia a escolha do melhor método conforme as dificuldades apresentadas pelo paciente.
Para melhor aproveitamento da terapia fonoaudiológica, a família deve ser orientada pelo fonoaudiólogo, esclarecendo a metodologia aplicada, os objetivos do SAC e por fim justificar a escolha do método relacionando ao paciente. "O Contexto familiar interfere diretamente no processo terapêutico podendo atrasar a construção do sujeito linguístico" ${ }^{15}$. Parte-se do pressuposto que o sujeito vai se constituir à medida que suas ações vão sendo interpretadas pelo outro através da internalização de papéis, definidos, inicialmente, pelas pessoas que são referência mais concreta da criança, como a família e, posteriormente, pelo grupo social maior no qual está inserida ${ }^{16}$. Sendo a família o primeiro núcleo social em que o individuo é inserido, esta é a primeira a buscar respostas e a lidar com as consequências de qualquer decisão para toda a vida ${ }^{17}$.

Todas as fontes bibliográficas pesquisadas são unânimes quanto ao não desenvolvimento da linguagem oral na $S A^{1-3,6}$. Pensando nisto torna-se essencial para os fonoaudiólogos, ampliar a visão sobre 0 atendimento prestado a essa população, que precisa de uma alternativa para expressar suas necessidades diárias, vontades e sentimentos.

Salienta-se também que, na literatura pesquisada, não foi encontrado até o término da pesquisa nenhum relato na tentativa de introduzir algum SAC na SA.

Tendo em vista os argumentos expostos, este trabalho tem como objetivo principal descrever o processo de introdução e desenvolvimento do uso do SAC na SA.

\section{APRESENTAÇÃO DO CASO}

Este trabalho caracterizou-se como um estudo de caso.

Fez parte deste estudo um portador da Síndrome de Angelman, Paciente nomeado como E., sexo feminino, 17 anos, que não possui linguagem oral estabelecida. De acordo com o depoimento inicial da mãe ocorrido em março de 2006, sua gestação e parto não apresentaram intercorrência. Ao nascer constatou-se alteração do perímetro cefálico, com valores menores do que o considerado normal, que se manteve até um ano, caracterizando a presença de microcefalia. O diagnóstico genético da SA ocorreu aos 12 anos de idade.

Em relação ao desenvolvimento motor, E. andou somente aos três anos. Atualmente apresenta uma marcha com base alargada, não realizando flexão de joelhos, contato do pé de forma espasmódica sem fase de apoio de calcanhar e fase de pulsão, transferindo rapidamente o peso.

E. faz uso de medicamento (Depakene $₫ 300 \mathrm{mg}$ ) para crises convulsivas. Com o uso, estas se 
tornaram esporádicas e caracterizam-se como convulsões tônico-clônica.

A paciente é dependente para atividades de vida diária (AVDs).

Quanto aos tratamentos, E. realizou terapia fonoaudiológica em outra instituição no período de 2002 a 2005. Este foi interrompido ao final do segundo semestre do ano de 2005, por fechamento da instituição. $O$ enfoque terapêutico até então utilizado com E. era o desenvolvimento da linguagem oral. Em março de 2006 iniciou atendimento na Clínica de Fonoaudiologia da Faculdade Estácio de Sá de Santa Catarina de junho de 2006, onde realizava terapia uma vez por semana com duração de 40 minutos no Estágio Supervisionado em Neurologia II.

E. frequenta a Associação de Pais e Amigos dos Excepcionais (APAE) do seu município desde 1992. A partir de 2006, iniciou atendimento fonoaudiológico em grupo, juntamente com os outros alunos da turma. Realizou atendimento com fisioterapeuta do nascimento até três anos e seis meses quando iniciou sua marcha. Após esse período, a terapia foi interrompida por apresentar comportamento pouco colaborativo involuntário.

Com o passar dos anos, seu desenvolvimento foi acompanhado por diversos profissionais como psicólogo, psiquiatra e psicopedagogo. Realizou também atendimento junto à equipe de Equoterapia.

Com relação aos atendimentos médicos, atualmente só realiza exames de rotina ou quando há problemas de saúde, principalmente junto as seguintes especialidades: ginecologista, neurologista, cirurgião dentista, ortopedista e otorrinolaringologista.

A participação da mesma na pesquisa ocorreu por meio da autorização do responsável que assinou o termo de consentimento livre e esclarecido e autorização de fotos e filmagens.

Segundo o relatório de avaliação inicial, E. apresentava alterações significativas quanto aos aspectos de linguagem e motricidade orofacial. $O$ objetivo da mãe em procurar o atendimento era melhorar a comunicação de sua filha tendo em vista que o surgimento da linguagem marca o desenvolvimento cognitivo e social da infância ${ }^{18}$, que neste caso estava extremamente atrasado.

As características de motricidade orofacial que E. apresentou foram: postura habitual de lábios entreabertos, língua protrusa e hipotônica, bochechas simétricas, palato duro normal, dentição permanente com 24 dentes em bom estado de conservação, baba presente em momentos de atividades direcionadas. Os outros aspectos, não foram possíveis avaliar devido a não colaboração da mesma.

Com relação às funções estomatognáticas, $\mathrm{E}$. mastiga os alimentos com a boca aberta, realiza movimentos verticalizados, amassamento dos alimentos com auxílio da língua, presença de ruídos constantes, interposição lingual no momento da deglutição, sobras em cavidade oral e escape de alimentos durante a mastigação. Não apresentou tosse nem engasgos durante a deglutição. Sua respiração é caracterizada como mista com predomínio oral.

$\mathrm{Na}$ avaliação de linguagem realizada por meio de situações de interação espontânea e de atividades lúdicas ${ }^{19}$, a paciente apresentou dificuldade de compreensão de ordens simples e complexas, dificuldade de concentração e atenção dirigida. Comunica-se através de linguagem não-verbal (gestos funcionais) e emissão de um único som contextualizado: /mã/ para mãe. Possui um déficit significativo da linguagem expressiva quanto aos aspectos de semântica, sintaxe, prosódia e fonologia caracterizando-se como ausência de fala funcional. Apresentou boa expressão facial conseguindo transmitir sentimentos de alegria e/ou tristeza. Possui intenção comunicativa presente em todos os momentos, demonstrando presença de aspectos pragmáticos.

Para coleta de dados foram utilizadas análises de todas as filmagens realizadas, totalizando oito trechos de terapia fonoaudiológica, com 11 minutos e 27 segundos. As terapias envolviam o brincar como estratégia para o desenvolvimento simbólico da criança ${ }^{20}$, ou seja, criar situação em que a criança se envolve processo de aprendizagem ${ }^{21}$. Estas filmagens foram realizadas em câmera digital Sony, modelo P-100, com periodicidade quinzenal no decorrer do primeiro semestre de 2007 durante os atendimentos fonoaudiológicos realizados no Estágio Supervisionado em Neurologia II, caracterizando uma pesquisa retrospectiva. A análise $e$ discussão de dados foram realizadas após a aprovação do Comitê de Ética. Além das gravações foi utilizado um diário de campo.

A partir das filmagens os trechos foram transcritos e apresentados durante a discussão em forma de diálogo, formalizando as situações contextualizadas ocorridas em terapia fonoaudiológica.

Os resultados foram descritos, analisados e interpretados. A apresentação dos resultados ocorreu em negrito e itálico representando os itens importantes observados durante a coleta.

A análise de dados foi realizada de forma qualitativa.

Os resultados foram apresentados e discutidos de acordo com o referencial teórico existente na área do tema pesquisado. Pode-se, no entanto, definir esse processo como uma sequência de atividades, que envolve descrição de dados, a categorização dos mesmos, sua interpretação e a redação do texto final. 
Este trabalho foi submetido e aprovado pelo Comitê de Ética em pesquisa com Seres Humanos da Faculdade Estácio de Sá de Santa Catarina sob o processo número 001/2007.

\section{RESULTADOS}

Os dados da avaliação foram descritos de forma a salientar os aspectos do desenvolvimento da linguagem, por ser o foco da pesquisa. Os resultados obtidos comparados à avaliação inicial serviram de parâmetros para importantes considerações.

Quanto ao desenvolvimento da linguagem, a paciente utiliza como meio de comunicação gestos funcionais e vocalizações de poucos sons isolados. Segundo relato de sua mãe, a paciente fala /mama/ desde um ano de idade. Esta palavra é utilizada não só para se dirigir à mãe, mas para todas as pessoas que ela tem carinho.

Ao término da avaliação, com base nos resultados descritos anteriormente, optou-se pelo desenvolvimento da linguagem verbal e não verbal por meio do uso de sistemas alternativos de comunicação, propiciando ao paciente uma forma alternativa de comunicação enquanto o oral não dá conta.

Após estudos dos sistemas alternativos de comunicação existentes, optou-se por uso do Picture Communication Symbols System (PCS) baseado no modelo de Mayer-Johnson Company (1990/2003).

Para seleção dos símbolos do PCS, foram utilizados centros de interesse, ou seja, objetos, atividades e comidas que segundo relato da mãe e babá, E. gostava muito. A inserção dos símbolos ocorreu de forma gradativa e lenta.

Os centros de interesses foram considerados atividades, jogos ou brincadeiras que traziam sensação de bem estar a paciente, fazendo com que sua motivação e participação nas sessões fonoaudiológicas se tornassem mais prazerosas.

A introdução dos símbolos do PCS foi apresentada a E. de maneira gradativa, ou seja, inicialmente, foi utilizado somente um símbolo por vez. A terapeuta realizava, primeiro, a apresentação do objeto concreto, por exemplo, bolacha (mostrava um pacote de bolacha e dava para paciente comer uma bolacha). Em seguida, para correlacionar o símbolo do PCS com o objeto trabalhado, os dois (objeto e símbolo) eram postos em cima da mesa correlacionando-os várias vezes.

Após realizar as duas etapas anteriores (apresentação e correlação) a terapeuta começou a introduzir a paciente ao uso do sistema. Para isso pegava a mão da mesma e colocava sobre o símbolo e simultaneamente entregava o objeto para $\mathrm{E}$. Este processo demorou duas sessões. Na terceira sessão essa etapa de apresentação, correlação e introdução não foram mais necessárias tendo em vista que $E$. nos atendimentos posteriores solicitava o objeto trabalhado por meio do apontar ou pegar o símbolo já trabalhado.

Vale salientar que a terapeuta durante o processo de estimulação da linguagem com a utilização dos símbolos do PCS também utilizava os recursos de comunicação alternativa enquanto falava.

Em síntese, o processo inicial foi composto pelas seguintes etapas: apresentação do objeto, correlação do objeto com o símbolo e introdução da paciente ao uso do sistema.

Com o passar das sessões foram introduzidos novos símbolos por categorias, como: comer, beber e brincar.

E. mostrou-se "perdida" frente aos símbolos novos, sendo iniciado os passos de apresentação do objeto, correlação do objeto com o símbolo e introdução da paciente ao uso do sistema.

A paciente negou as categorias apresentadas direcionando sua escolha para os símbolos que já Ihe eram familiares, como os de bolacha, água e balão. A terapeuta a cada solicitação da paciente, por exemplo, bolacha, direcionava sua mão para o símbolo de comer e depois para o símbolo de bolacha, fazendo simultaneamente a correlação com a categoria e oralização da estrutura sintática completa dos símbolos apresentados, por exemplo: "Eu quero comer bolacha". O objetivo neste momento era ampliação da estruturação dos aspectos sintáticos da linguagem por meio do uso do PCS.

A inserção de novos símbolos aconteceu de maneira lenta e gradativa. Alguns símbolos foram melhor reconhecidos pela paciente (pentear o cabelo) outros foram totalmente ignorados (fotos) como na situação.

Percebe-se, ao final dos atendimentos, que E., devido ao comprometimento cognitivo e hiperatividade, relacionou alguns símbolos utilizando-os de forma funcional. A mesma apresentou durante os atendimentos, em determinados momentos, inconsistência de respostas.

Dos 15 símbolos trabalhados, adquiriu funcionalidade apenas para cinco símbolos.

Vale salientar que foram realizadas 12 sessões de atendimentos fonoaudiológicos com este objetivo, demonstrando resultados satisfatórios devido ao curto período de atendimento, sendo este realizado uma vez por semana, com duração de 40 minutos, totalizando 10 horas de atendimento.

Durante a realização desta pesquisa foi possível observar nitidamente à dificuldade de trabalhar com outras atividades fonoaudiológicas simultaneamente a introdução e desenvolvimento da comunicação alternativa. 
Algumas das características da SA que dificultaram a introdução do sistema foram o déficit cognitivo e a agitação constante. A paciente possui dificuldade de atenção e concentração e movimenta-se pela sala a maior parte do período trabalhado.

Em vários momentos foi possível observar também que a paciente apresentou a não confirmação dos símbolos pedidos deixando de lado ou recusando o objeto concreto que era dado a mesma pela terapeuta quando solicitado pela paciente.

Com aumento das cartelas dos símbolos do PCS trabalhados (divididos por categorias) foi difícil a utilização do sistema sendo alguns símbolos foram ignorados pela paciente como fotos.

\section{DISCUSSÃO}

As seis características da SA prevalentes entre os portadores são: retardo mental severo, ausência da fala, marcha espástica, incitabilidade, hiperatividade, sorrisos frequentes sem motivos e presença de diastemas na arcada dentária ${ }^{1-6}$. $O$ atraso da linguagem ocorre entre seis e 12 meses.

Para um diagnóstico preciso da SA é necessário embasamento em critérios clínicos e provas de laboratórios ${ }^{5}$, sendo geralmente realizado tardiamente a medida que as crianças crescem ${ }^{1,2}$. E. foi diagnosticada somente aos 12 anos de idade confirmando os dados encontrados na literatura. Vale salientar também a necessidade de um trabaIho interdisciplinar onde a relação de reciprocidade entre os profissionais proporcionaria o diálogo entre as diversas disciplinas envolvidas levando a interação ${ }^{22,23}$ e consequentemente a melhora das possibilidades de desenvolvimento dos pacientes.

E. parece possuir compreensão suficiente para se comunicar. Porém, segundo a literatura, mesmo no mais alto nível de desenvolvimento a linguagem não se desenvolve. A capacidade de linguagem não-verbal varia muito sendo que os indivíduos com melhor desenvolvimento são capazes de aprender alguns sinais e usá-los como ajuda de expressão juntamente com outros meios de comunicação baseados em imagens ${ }^{24}$.

Com base na avaliação foi realizado um planejamento terapêutico em que seria estimulada simultaneamente a linguagem oral ao uso de sistemas alternativos de comunicação. "Em outras palavras, os procedimentos utilizados na comunicação alternativa não estariam competindo com a fala, mas dando suporte, apoio e, em última hipótese, sendo alternativa a ela" ${ }^{25}$. As habilidades para a fala que o indivíduo possa ter ou vir a apresentar com estes sistemas não são inibidas, pois este sistema proporciona a eliminação de uma possível tensão existente no ato de comunicação, possibilitando a emissão oral, acompanhada pela indicação do símbolo desejado ${ }^{26}$. O contexto terapêutico baseado em recursos e estratégias de comunicação suplementar valorizava, além dos aspectos verbais, os recursos para-linguísticos não verbais, como os gestos representativos ${ }^{27}$.

A terapia proposta foi individual para um melhor controle das atividades propostas, realizando também fisioterapia. Autores relatam que o atendimento deve ser individual e de caráter multidisciplinar com os seguintes profissionais: fonoaudiólogo, fisioterapeuta e terapeuta ocupacional ${ }^{2,5}$.

O trabalho do fonoaudiólogo deve visar o aumento da capacidade pulmonar (respiratória), deve-se trabalhar também: sucção, mastigação, deglutição (atípica), praxias e expressões faciais. Também se pode trabalhar a estimulação de uma forma geral ${ }^{5}$.

A comunicação alternativa foi escolhida por ser um conjunto de procedimentos técnicos e metodológicos utilizado por pessoas que são acometidas por alguma doença, deficiência ou alguma situação permanente ou temporária que impeça o indivíduo de falar ${ }^{25}$, não sendo um método de alfabetização e de inibição da fala ${ }^{28}$.

Comunicação Suplementar e/ou Alternativa é conceituada como todas as formas existentes de comunicação que não utilizam ou utilizam de forma ineficiente a linguagem oral, seja por qualquer alteração que impeça de adquirir a fala ${ }^{29}$, ou por pessoas que adquiriram a fala, mas que por alguma razão não conseguem mais se comunicar pela linguagem oral sendo utilizada na prática clínica para compensar temporariamente ou permanentemente incapacidades e alterações severas na fala, comunicação expressiva, linguagem e escrita ${ }^{25,29}$.

Os sistemas suplementares e/ou alternativos de comunicação têm como objetivo fundamental suprir a limitação ou falta da linguagem oral e seu objetivo principal é fornecer ou facilitar um canal comunicativo assegurando o desenvolvimento cognitivo, afetivo e de linguagem ${ }^{30}$.

Considera-se comunicação suplementar quando o indivíduo utiliza um outro meio de comunicação para compensar deficiências que a fala apresenta, mas sem substituí-la totalmente. Já a comunicação alternativa é utilizada quando o indivíduo utiliza um outro meio de comunicação para se comunicar ao invés da fala, devido à impossibilidade de articular ou produzir sons adequadamente.

Os principais candidatos ao uso dos sistemas complementares de comunicação alternativa são: pessoas que possuem compreensão preservada e dificuldade de expressão, pessoas que possuem fala ininteligível, distúrbios graves como: autismo e agnosia auditiva, paralisia cerebral, traumatismo 
cranianos (TCE) (para os casos que atingiram regiões cerebrais responsáveis pela fala ou linguagem, motor e cognitivo), doenças degenerativas, afasias e pessoas que possuem dificuldade ou impedimento na movimentação dos órgãos fonoarticulatórios (traumatismos nas cordas vocais) ${ }^{25}$.

No Brasil, o uso da comunicação alternativa é muito novo, cujo sistemas mais usados: Blissynbolics System (BLISS), o Picture Comunication Symbols Sistema Pictográfico de comunicação (PCS), e com menos uso o Pictogram Ideogram Ccommunication System ( PIC) ${ }^{31}$.

O PCS foi selecionado para E. por apresentar, de todos os sistemas estudados, os símbolos que mais se relacionam com os objetos reais, sendo sua visualização e compreensão mais simples. Além disso, pode ser utilizado de forma simples e rápida, podendo ser manipulado de acordo com a necessidade de cada paciente ${ }^{32}$. Os desenhos do PCS são mais apropriados em casos de vocabulário limitado, estruturas de frases curtas e nível de linguagem simples ${ }^{28}$.

O PCS foi idealizado por Roxana Mayer Johnson. É o sistema mais utilizado atualmente composto por um código pictográfico, caracterizando-se por desenhos e símbolos, inicialmente com 709 figuras, incluindo números e letras do alfabeto ${ }^{32,33}$. Este é de fácil compreensão e aquisição. Além das figuras que compõem este sistema pode-se utilizar fotos, figuras de revistas, palavras escritas ou combinação de ambas ${ }^{28,30}$, caracterizando-se como um sistema aberto. O PCS apresenta atualmente tem cerca de 3.000 figuras que expressam uma variedade de palavras usadas em situações e atividades de vida diária ${ }^{34}$.

O PCS é primeiramente composto de linha simples. A palavra que cada desenho simboliza está impressa em cima do desenho. Também estão incluídos o alfabeto, os números e os círculos para cores ${ }^{35}$.

Os símbolos do PCS são divididos em seis categorias, cada uma das quais é recomendável que apresente cores diferentes: na cor amarela devemse utilizar pessoas e pronomes pessoais, na cor verde verbos, na cor azul adjetivos e advérbios, na cor laranja nomes correspondentes a varias categorias como: comida, objetos, animais entre outros, classificadas como substantivos, na cor branca para termos diversos como: letras do alfabeto, cores, números, classificados como miscelânia, e na cor rosa, palavras curtas que são utilizadas no dia a dia, consideradas palavras e/ou frases prontas sociais 29,36 . Estas categorias foram criadas para facilitar a identificação e a localização do símbolo que deseja ser usado ${ }^{32,33}$.
Além disso, os símbolos devem ser dispostos na prancha de comunicação na seguinte ordem: rosa, amarelo, verde, laranja e azul, que condiz com a ordem sintática de uma frase. A categoria que utiliza a cor branca pode ser disposta em qualquer posição, Você cumprimenta uma pessoa (categoria rosa), se coloca no discurso (categoria amarela), utiliza o verbo (categoria verde), completa a frase com substantivos (categoria laranja) e adjetivos (categoria azul).

Inicialmente os símbolos são usados soltos. Com E. a inserção de novos símbolos aconteceu de maneira lenta e gradativa. A estimulação de linguagem com auxílio de símbolos ocorreu da forma mais natural possível. O indivíduo que está aprendendo a usar o recurso de comunicação alternativa deve ter simultaneamente o estímulo falado e a interação com um parceiro relata.

A codificação por cor da categoria utilizada pode ser realizada por duas formas diferentes. Uma forma é copiar os símbolos em diferentes tons de papel colorido. A outra forma é imprimir os símbolos coloridos em papel branco. A mesma completa que o PCS deve ser personalizado sempre que necessário. A personalização pode ser feita à mão ou no computador utilizando programa Boardmaker ${ }^{35}$. Nas pranchas de comunicação futuramente serão dispostas figuras do cotidiano do usuário fazendo com que os mesmos possam expressas suas vontades, ideias e sentimentos ${ }^{31,37}$.

Para E. foram apresentados no primeiro atendimento os dois modelos sendo que o modelo impresso colorido no fundo branco chamou mais a atenção, sendo este utilizado nos símbolos posteriores.

O aumento do número de símbolos ocorreu de acordo com a necessidade das atividades propostas e com a sistematização dos símbolos anteriores pela paciente.

Os símbolos devem ser usados na medida do necessário, em conjunto com outras fotos, desenhos ou outros conjuntos de símbolos.

As principais dificuldades encontradas neste processo foram com relação às características da própria síndrome, sendo elas, hiperatividade, dificuldade de compreensão devido à deficiência mental e dificuldade de inserção de atividades paralelas ao trabalho e introdução e desenvolvimento da comunicação alternativa.

Vale salientar que o tempo é um fator primordial para o desenvolvimento de trabalhos com SAC. Considera-se ao término desta pesquisa que E., apesar do curto período de tempo de atendimento, apresentou resultados satisfatórios quanto ao uso do PCS. 
Nesta perspectiva, para finalizar, ressalta-se que a comunicação alternativa adquire um significado terapêutico reabilitativo e que é possível encontrar em cada pessoa com deficiência verbal algum meio de comunicação, não só para receber informações ou aprendizados, mas, sobretudo, para exprimir sentimentos e desejos ${ }^{33}$.

\section{CONCLUSÃO}

A SA tem como uma das principais características fonoaudiológicas ausência de fala funcional ou um desenvolvimento de linguagem oral restrito. Sendo o fonoaudiólogo o profissional preparado para estar reabilitando este paciente com estas alterações, cabe ressaltar a importância de ampliar sua atuação com outros métodos, por exemplo, sistemas de comunicação alternativa.
O processo de introdução e desenvolvimento do uso de SAC foi composto pelas seguintes etapas: apresentação do objeto, correlação do objeto com o símbolo e introdução da paciente ao uso do sistema utilizando sempre centros de interesse da paciente. A escolha do sistema a ser utilizado deve ser realizada com bastante cuidado a fim de propiciar ao paciente o método mais adequado as suas alterações.

Com esta pesquisa fica claro que o Fonoaudiólogo é capaz de estar estimulando a linguagem verbal e não-verbal com auxílio de técnicas específicas chamadas SAC para estar auxiliando ou apoiando os pacientes diagnosticados com SA. Estes podem servir de apoio para desenvolverem um meio comunicativo e consequentemente uma melhora na interação social.

\begin{abstract}
Background: Syndrome of Angelman (SA) and alternative communication. Procedures: with the objective to describe the process for introducing and developing the use of an Alternative Communication System(SAC) in the SA, we used a case study, carried through in the Clinic of Speech Therapy of Faculdade Estácio de Sá of Santa Catarina. E., diagnosed with SA, 17year old, without verbal language. Collection of data carried through with 8 stretches of therapies recorded during the first half of 2007 and notations in the field daily record. Results: the program of communication chosen was the Picture Communication Symbols System (PCS). For electing the symbols, we used interest centers of the patient. The process for inserting symbols happened in a slow and gradual manner. The therapist carried through the presentation of the concrete object, after that, she correlated the symbol with the object. Both were placed on top of the table correlating them several times. For introducing the patient to the use of the system, the therapist took the hand of the patient and placed it on the symbol and simultaneously delivered the object. With the passing of the sessions, new symbols were introduced. E. revealed being "lost" denying the presented categories, directing its choice for the symbols that already were familiar. Of the 15 worked symbols, 5 symbols acquired functionality. Conclusion: SAC becomes a feasible communication mode for SA. The process of introducing and developing SAC use was made up by: presentation, correlation and introduction of the patient to the use of the system. We stress out that this is a slow work that demands participation of the family.
\end{abstract}

KEYWORDS: Angelman Syndrome; Communication; Language

\section{REFERÊNCIAS}

1. Artigas-Pallarés J, Brun-Gasca C, Gabau-Vila E, Guitart-Feliubadaló M, Camprubi-Sanches C. Aspectos médicos y conductuales del síndrome de Angelman. Rev Neurol. 2005; 41(11):649-56.

2. Queiroz AM, Bottós GN, Pin NAM, Lessa FCR, Silva RAB, Freitas AC. Síndrome de Angelman: relato de caso clínico. Rev Inst Ciênc Saúde. 2005; 23(3):235-8.
3. Sanvito WL. Síndromes neurológicas. 2. ed. São Paulo: Atheneu; 1997. 599 p.

4. Cerda MM, Gago RB. Características clínicas de dos probables casos de Síndrome de Angelman en el Hospital Nacional de Niños. Acta Méd. 2004; 46(2):88-90.

5. Garrido YJ. El Síndrome de Angelman. Avances Pediátricos. 2005; 29(3):35-8.

6. Valente KDR. Angelman syndrome: characteristics of epilepsy, electroencephalographic abnormalitties and correlaction to genetic mechanisms. [tese] Arq 
Neuro-Psiquiatr. 2002; 60(4). Disponível em: URL: http://www.scielo.br/scielo.php?script=sci_arttext\& pid=S0004-282X2002000600035\&lng=pt\&nrm=iso \&tlng=en. Acesso em 27 set 2006.

7. Cupello RCM. 1000 perguntas em fonoaudiologia. Rio de Janeiro: Revinter; 2004. 263 p.

8. Rodrigues PMT, Carvalho ELL, Areias AM, Burguetti FAC, Wendling FF, Sabaté FM, et al. Incidência de distúrbios da comunicação humana em pré-escolares. Temas sobre Desenvolv. 2000; 9(50):24-7.

9. Mandrá PP. Retardo de linguagem. Temas sobre desenvolvimento. 2008; 16(91):34-7.

10. Tetzchner SV, Brekke KM, Sjothun B, Grindheim E. Inclusão de crianças em educação pré-escolar regular utilizando comunicação suplementar e alternativa. Rev Bras Educ Espec. 2005; 11(2): 151-84.

11. Azevedo TFP. Educação da pessoa com necessidades especiais: o caso de Juiz de Fora. Temas sobre Desenvolv. 2000; 9(50):40-7.

12. Amaral LA. Alguns apontamentos para reflexão/ discussãosobre:diferença/deficiência/necessidades educacionais especiais. Temas sobre Desenvolv. 1999; 8(47):17-23.

13. Marques CA. Revisão do conceito de normalização em educação especial. Temas sobre desenvolvimento. 1999. 8(44):16-21.

14. Ramos AS, Alves LM. A Fonoaudiologia na relação entre escolas regulares de ensino fundamental e escolas de educação especial no processo de inclusão. Rev Bras Educ Espec. 2008; 14(2):235-50.

15. Tavares CJR, Madalozzo D, Ferraz e Ferraz MGC. Interferências da família na produção de distúrbios de linguagem. Temas sobre o Desenvolv. 2000. 9(49):16-22.

16. Leite GA, Monteiro MIB. A construção da identidade de sujeitos deficientes no grupo terapêutico-fonoaudiológico. Rev Bras Educ Espec. 2008; 14(2):189-200.

17. Cavalcante F. Família, subjetividade e linguagem: gramáticas da criança "anormal". Ciênc Saúde Coletiv. 2001; 6(1):125-37.

18. Coelho ACC, Lemma EP, Lopes-Herrera SA. Relato de caso: privação sensorial de estímulos e comportamentos autísticos. Rev Soc Bras Fonoaudiol. 2008; 13(1): 75-81.

19. Silva RA, Lopes-Herrera SA, De Vitto LPM. Distúrbio de linguagem como parte de um transtorno global do desenvolvimento: descrição de um processo terapêutico fonoaudiológico. Rev Soc Bras Fonoaudiol. 2007; 12(4):322-8.

20. Batista CVM. A importância do brincar para a emergência da função simbólica. Temas sobre Desenvolv. 2008; 16(93):136-41.
21. Enumo SRF. Avaliação assistida para crianças com necessidades educacionais especiais: um recurso auxiliar na inclusão escolar. Rev Bras Educ Espec. 2005; 11(3):335-54.

22. Graciano MIG, Figueira E. A deficiência: aspectos sociais da reabilitação e trabalho interdisciplinar. Temas sobre Desenvolv. 2000; 9(49):40-51.

23. Saupe R, Budó MLD. Pedagogia interdisciplinar: "educare" (educação e cuidado) como objeto fronteiriço em saúde. Texto Contexto Enferm. 2006; 15(2):326-33.

24. Thompson MW, Mcinnes RR, Willard HF. Thompson: genética humana. 5. ed. Rio de Janeiro: Guanabara Koogan; 1993.

25. Manzini JE. Conceitos básicos em comunicação alternativa e suplementar. In: Kester C, organizador. Educação, universidade e pesquisa. Marília: UNESP; 2001. p. 163-78.

26. Almeida MA, Piza MHM, Lamônica DAC. Adaptações do sistema de comunicação por troca de figuras no contexto escolar. Pró-Fono. 2005; 17(2):233-40.

27. Guarda NS, Deliberato D. Caracterização dos enunciados de um aluno não-falante usuário de recurso suplementar de comunicação durante a construção de histórias. Rev Bras Educ Espec. 2006; 12(2):269-88.

28. Silva CM, Reis NMM. Utilização da comunicação suplementar e/ou alternativa na paralisia cerebral. In: Fonseca LF, Lima CL. Paralisia cerebral: neurologia, ortopedia, reabilitação. Rio de Janeiro: Guanabara Koogan; 2004. p. 281-97.

29. Moreira EC, Chun RYS. Comunicação suplementar e/ou alternativa: ampliando a possibilidade de indivíduos sem fala funcional. In: Lacerda CBF, Panhoca I. Tempo em fonoaudiologia. São Paulo: Universitária; 1997. p. 37-175.

30. Panhan H, Fernandes AS. Comunicação suplementar e/ou alternativa. 7f. [apostila] Curso de Comunicação Suplementar e Alternativa. Grupo de Estudos em Sistemas Suplementares e/ou Alternativos de Comunicação, GESSAC, São Paulo; 1999.

31. Trevizor TT, Chun RYS. O desenvolvimento da linguagem por meio do sistema pictográfico de comunicação. Pró-Fono. 2004; 16(3):323-32.

32. Rossel C, Basil C. Sistemas de sinais manuais e gráficos: características e critérios de uso. In: Almirall CB, Soro-Camats E, Bultó CR. Sistemas de sinais e ajudas técnicas para a comunicação alternativa e a escrita: princípios teóricos e a aplicações. São Paulo: Santos; 2003. p. 7-19.

33. Guanella D. E se falta a palavra qual a comunicação, qual a linguagem? Discurso sobre a comunicação alternativa (segunda parte). São Paulo: Memnon; 1999. 
34. Almeida MA, Piza MH, Lamonica DA. [Adaptation of the picture exchange communication system in a school context]. Pró-Fono. 2005; 17(2):233-40.

35. Mayer-Johnson RM. Guia dos símbolos de comunicação pictográfica. Porto Algre: Clik; 1998.

36. Basil C. Sistemas aumentativos e alternativos de comunicação. In: Casanova JP. Manual de fonoaudiologia. 2. ed. São Paulo: Artmed; 1997. p. 338-47.

37. Chun RYS. Comunicação suplementar e/ou alternativa: favorecimento da linguagem de um sujeito não falante. Pró-Fono. 2003; 15(1):55-64.

DOI: 10.1590 / S1516-18462009005000033

RECEBIDO EM: 12/12/2007

ACEITO EM: 25/05/2008

Endereço para correspondência:

Melissa Watzko Eskelsen

Av. Governador Adolfo Konder, 251/304

São José - SC

CEP: 88101-400 